\title{
WEIGHTED JOIN-SEMILATTICES AND TRANSVERSAL MATROIDS
}

BY

\author{
RICHARD A. BRUALDI( 1 )
}

ABSTRACT. We investigate join-semilattices in which each element is assigned a nonnegative weight in a strictly increasing way. A join-subsemilattice of a Boolean lattice is weighted by cardinality, and we give a characterization of these in terms of the notion of a spread. The collection of flats with no coloops (isthmuses) of a matroid or pregeometry, partially ordered by set-theoretic inclusion, forms a join-semilattice which is weighted by rank. For transversal matroids these join-semilattices are isomorphic to join-subsemilattices of Boolean lattices. Using a previously obtained characterization of transversal matroids and results on weighted join-semilattices, we obtain another characterization of transversal matroids. The problem of constructing a transversal matroid whose join-semilattice of flats is isomorphic to a given join-subsemilattice of a Boolean lattice is then investigated.

1. Introduction. We are motivated by a recent study [3] of transversal matroids in which a characterization of transversal matroids is given in terms of the joinsemilattice of flats with no coloops (isthmuses). The characterization depends on an algorithm used to construct a family of flats. We consider here the more general situation of a join-semilattice $J$ in which each element has assigned to it a nonnegative weight where the only assumption is that weight is a strictly increasing function on $\mathrm{J}$. We introduce the concept of a spread for such a weighted join-semilattice, show a spread is unique if it exists at all, and give a characterization of join-semilattices of a finite Boolean lattice which are weighted by cardinality. Making use of the characterization of transversal matroids given in [3], we then give an alternate characterization which has the advantage of not depending on an algorithm. Finally we consider the problem of constructing a transversal matroid such that its weighted join-semilattice of flats with no coloops is isomorphic to a given join-subsemilattice of a Boolean lattice.

A family of objects differs from a set in that the objects may be repeated. We use parentheses ( ) to denote families and braces \{\} to denote sets. If $E$ is a

Received by the editors August 31, 1972.

AMS (MOS) subject classifications (1970). Primary 05B35, 06A20; Secondary 05A05.

Key words and phrases. Weighted semilattice, spread, Boolean lattice, transversal matroid.

(1) Research partially supported by National Science Foundation Grant No. GP. 17815 . 
set and $\mathcal{U}=\left(a_{i}: i \in I\right)$ is a family of elements of $E$, then for $x \in E$ we define the multiplicity of $x$ in $\mathbb{Q}$ by

$$
m(\mathscr{U}, x)=\left|\left\{i \in I: a_{i}=x\right\}\right| \text {. }
$$

This multiplicity may be 0 . If $\mathfrak{U}_{1}, \mathfrak{U}_{2}$ are two families of elements of $E$, we write $\mathfrak{U}_{1} \leq \mathscr{U}_{2}$ provided $m\left(\mathscr{U}_{1}, x\right) \leq m\left(\mathscr{U}_{2}, x\right)$ for all $x \in E$. We consider two families to be identical if $m\left(\mathscr{U}_{1}, x\right)=m\left(\mathscr{U}_{2}, x\right)$ for all $x \in E$. The cardinality $\|\mathscr{U}\|$ of a family $\mathscr{Q}$ is defined by $\|\mathscr{U}\|=|I|$.

2. Weighted join-semilattices. A join-semilattice is a partially ordered set $J$ such that each pair $a, b$ of elements of $J$ has a least upper bound, which is denoted by $a \vee b$. A nonempty collection of subsets of a set $E$ which is closed under union is a join-semilattice; the partial order is set-theoretic inclusion. Such a join-semilattice is a join-subsemilattice of the Boolean lattice $B(E)$ of all subsets of $E$. A finite join-semilattice has a maximal element which we usually denote by 1 ; it need not have a minimal element but, if it does, it is usually denoted by 0 . It is well known that a finite join-semilattice with a minimal element is a lattice [1].

If $P$ is a partially ordered set, then a mapping $\omega$ from $P$ to the nonne gative integers is a weighting of $P$ if $a<b$ implies $\omega(a)<\omega(b)(a, b \in P)$. A weighting of $P$ need not be a grading [1] of $P$, for we do not assume that $\omega(b)=\omega(a)+1$ if $b$ covers $a$. If $E$ is a set and $P$ is a collection of subsets of $E$ partially ordered by set-theoretic inclusion, then $\omega(A)=|A|$ defines a weighting of $P$. A partially ordered set with a specific weighting is called a weighted partially ordered set.

Let $P$ be a weighted partially ordered set with a maximal element 1 and set $\omega(1)=r$. Thus any chain has length at most $r$. A spread of $P$ is a family $\mathscr{U}=$ $\left(a_{i}: i \in I\right)$ of elements of $P$ such that the following condition holds: if $x \in P$ with $\omega(x)=r-k(0 \leq k \leq r)$, then

$$
\left|\left\{i \in I: a_{i} \geq x\right\}\right|=k \text {. }
$$

In other words, exactly $k$ members of $\mathcal{Q}$ are greater than or equal to $x$ if $\omega(x)=$ $r-k$. If $P$ has a minimal element 0 , then clearly the cardinality $\|$ $2 \|$ of the spread $\mathcal{U}$ equals $r-\omega(0)$. Thus if $\omega(0)=0$, a spread of $P$ has exactly $r=\omega(1)$ elements. The notion of a spread arose in a characterization of transversal matroids [3], which will be fully explained in due course.

Theorem 2.1. If the weighted partially ordered set $P$ with maximal element bas a spread, the spread is unique.

Proof. Suppose $\mathcal{O}$ is a spread of $P$, and let $\omega(1)=r$. Let $a \in P$ with $\omega(a)=$ $r-k$. Then from $(2.0 .1)$ we conclude that the multiplicity of $a$ in $\mathfrak{Q}$ is given by 


$$
m(\mathscr{U}, a)=k-\sum_{x>a} m(\mathscr{U}, x) \text {. }
$$

This implies, in particular, that $\Sigma_{x>a} m(\mathbb{R}, x) \leq k$. The equation (2.1.1) along with $m(\mathscr{U}, 1)=0$ furnishes a recursion formula for the multiplicities of elements of $P$ in $\mathcal{U}$. Since these multiplicities are uniquely determined, so is the spread U.

The proof of Theorem 2.1 gives an algorithm for determining a spread if one exists. If there is no spread, then there is some element $a$ of $P$ with $\omega(a)=r-k$ such that $\Sigma_{x>a} m(\mathbb{U}, x)>k$.

The next theorem furnishes examples of weighted partially ordered sets which have spreads. Theorem 2.3 will then show that these examples are not very special.

Theorem 2.2. Let $\mathrm{J}$ be a join-subsemilattice of the Boolean lattice on a set $E$ with $|E|=r$. Suppose $E$ is the maximal element of $J$, and let $J$ be weighted by the cardinality function. Then

$$
\begin{aligned}
& J \text { has a spread } \mathscr{U} \text {, and }\|\mathrm{U}\|=r-|\cap\{A: A \in J\}| . \\
& m(\mathscr{U}, A)=|(\bigcap X: A \varsubsetneqq X \in J)|-|A| \quad(A \in J) .
\end{aligned}
$$

Proof. The assumption that $E$ is the maximal element of $J$ is one of convenience. If this were not the case, $E$ would be replaced by a smaller set.

Let $A \in J$ with $|A|=r-k$. We need to show that $m(2 l, A)=k-$ $\Sigma_{A \subsetneq X \in J} m(\mathbb{R}, X)$ and $m(\mathbb{R}, E)=0$ allows us to define $\mathcal{U}$ recursively. This is surely true for $k=0$ (that is, $A=E$ ). We proceed by induction on $k$. Let $A \in J$ with $|A|=r-k$. Let $X_{1}, \cdots, X_{t}$ be the members of $J$ which strictly contain $A$. Thus $\left|X_{i}\right|=r-k_{i}$ where $k_{i}<k(1 \leq i \leq t)$. Since $J$ is a join-semilattice, $X_{i_{1}} \cup$ $\cdots \cup X_{i_{s}} \in J$ whenever $1 \leq i_{1}<\cdots<i_{s} \leq t$; let $\left|X_{i_{1}} \cup \cdots \cup X_{i_{s}}\right|=r-k_{i_{1}} \ldots i_{s}$ where $k_{i_{1}} \ldots i_{s}<k$. Thus by induction the recursion has produced $k_{i_{1}} \ldots i_{s}$ members of 2 that contain $X_{i_{1}} \cup \cdots \cup X_{i_{s}}$. Hence by the principle of inclusionexclusion exactly

$$
n=\sum_{1 \leq i \leq t} k_{i}-\sum_{1 \leq i_{1}<i_{2} \leq t} k_{i_{1} i_{2}}+\sum_{1 \leq i_{1}<i_{2}<i_{3} \leq t} k_{i_{1} i_{2} i_{3}}-\cdots
$$

members of 2 that strictly conta in $A$ have been produced. But 


$$
\begin{aligned}
n= & \sum_{1 \leq i \leq t}\left|E \backslash x_{i}\right|-\sum_{1 \leq i_{1}<i_{2} \leq t}\left|E \backslash\left(x_{i_{1}} \cup X_{i_{2}}\right)\right| \\
& +\sum_{1 \leq i_{1}<i_{2}<i_{3} \leq t}\left|E \backslash\left(X_{i_{1}} \cup X_{i_{2}} \cup X_{i_{3}}\right)\right|-\cdots \\
= & \left|\bigcup_{i=1}^{t}\left(E \backslash X_{i}\right)\right|=\left|E \backslash \bigcap_{i=1}^{t} x_{i}\right|=r-\left|\bigcap_{i=1}^{t} x_{i}\right| .
\end{aligned}
$$

Since $A \subseteq X_{i}(1 \leq i \leq t), A \subseteq \bigcap_{i=1}^{t} X_{i}$ so that $\left|\bigcap_{i=1}^{t} X_{i}\right| \geq r-k$ and $n \leq r-$ $(r-k)=k$. Thus we can define $m(\mathscr{l}, A)$ by $k-n \geq 0$. But $k-n=k-$ $\left(r-\left|\bigcap_{i=1}^{t} X_{i}\right|\right)=\left|\bigcap_{i=1}^{t} X_{i}\right|-(r-k)=\left|\bigcap_{i=1}^{k} X_{i}\right|-|A|$. Thus we have proved that $J$ has a spread and that $(2.2 .2)$ is satisfied.

We have yet to prove that $\|\mathfrak{U}\|=r-|\bigcap\{A: A \in J\}|$. This is surely true if $\varnothing \in J$. If $\varnothing \notin J$, then $J^{*}=J \cup\{\varnothing\}$ is a join-subsemilattice of the Boolean lattice on $E$ and has a spread $\mathfrak{I}^{*}$ where $\left\|\mathbb{R}^{*}\right\|=r$. But

$$
\|\mathscr{U}\|=\left\|\mathscr{U}^{*}\right\|-m\left(\mathscr{U}^{*}, \phi\right)=r-|(\cap X: X \in J)|
$$

according to (2.2.2), and this establishes the formula for $\|$ et $\|$.

Corollary 2.3. If $\mathcal{U}=\left(A_{i}: i \in I\right)$ is the spread of the join-subsemilattice $J$ of the Boolean lattice on $E$, then for $A \dot{\epsilon} J$,

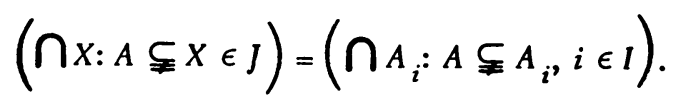

Proof. The set on the right side of (2.3.1) surely contains that on the left. Suppose now that $A \varsubsetneqq X \in J$ but $X \neq A_{i}(i \in I)$. Then arguing by induction ( $|X|>|A|)$, and using (2.2.2) we conclude that

$$
X=(\cap Y, X \varsubsetneqq Y \in J)=\left(\bigcap_{A_{i}}: X \varsubsetneqq A_{i}, i \in I\right) .
$$

Thus if $A \varsubsetneqq X \in J$, then either $X=A_{i}$ for some $i \in I$ or else there is $J \subseteq I$ such that $X=\bigcap_{i \in J} A_{i}$. Since $A \varsubsetneqq X, A \varsubsetneqq A_{i}(i \in J)$ and this establishes (2.3.1).

Consider the join-semilattice $J$ of Theorem 2.2 and its spread $\mathscr{U}=$ $\left(A_{i}: i \in I\right)$. Let $A \in J$ and let $\mathscr{U}_{A}$ be the subfamily of $\mathscr{U}$ consisting of all members $A_{i}$ of $\mathscr{V}_{\text {with }} A \subseteq A_{i}$. $\mathscr{U}_{A}$ is the spread of the interval $[A, E]$ of $J$. Then for $A, B \in J, A \subseteq B$ if and only if $\mathscr{U}_{B} \leq \mathscr{U}_{A}$. For if $A \subseteq B$, then surely $\mathscr{U}_{B} \leq \mathscr{U}_{A}$. On the other hand if $\mathfrak{U}_{B} \leq \mathfrak{U}_{A}$, then $B \in \mathfrak{U}_{B}$ implies $B \in \mathfrak{U}_{A}$ so that $A \subseteq B$, while $B \notin \mathbb{U}_{B}$ implies $B \notin \mathscr{U}$ (i.e. $m(\mathscr{U}, B)=0$ ), which by $(2.2 .2)$ and (2.3.1) implies 


$$
B=\bigcap\left(A_{i}: A_{i} \in \mathscr{V}_{B}\right) \supseteq \bigcap\left(A_{i}: A_{i} \in \mathscr{V}_{A}\right) \supseteq A .
$$

Thus $A \subseteq B$. Hence the partial order of $J$ is determined by the partial order on the $\mathscr{U}_{A}(A \in J)$.

If $J_{1}$ and $J_{2}$ are two join-semilattices, an injection $\sigma: J_{1} \rightarrow J_{2}$ is a semilattice monomorphism if $\sigma(a \vee b)=\sigma(a) \vee \sigma(b)$ for all $a, b \in J_{1}$. We shall be interested now in weighted semilattices which are isomorphic where the isomorphism preserves weights.

Theorem 2.4. Let J be a weighted join-semilattice with $\omega(1)=r$. Let $E$ be a set with $|E|=r$. Then there is a semilattice monomorphism $\sigma: J \rightarrow B(E)$, the Boolean lattice on $E$, such that $\omega(a)=|\sigma(a)|$ for all $a \in J$ if and only if $J$ bas a spread of at most $r$ elements.

Proof. By Theorem 2.2 if such a $\sigma$ exists, $J$ has a spread with at most $r$ elements. Suppose now $J$ has a spread $\mathscr{U}=\left(a_{i}: i \in I\right)$ with $\|\mathscr{R}\|=|I| \leq r$. For $a \in J$, let $I_{a}=\left\{i \in I: a_{i} \geq a\right\}$. Thus if $\omega(a)=r-k,\left|I_{a}\right|=k$. We define a map $r: J \rightarrow$ $B(I)$ by $\tau(a)=I \backslash I_{a}$. Thus for $a, b \in J, \tau(a \vee b)=\lambda I_{a \vee b}$. But $I_{a \vee b}=I_{a} \cap I_{b}$; for if $a_{i} \geq a \vee b$, then $a_{i} \geq a, b$ so that $I_{a \vee b} \subseteq I_{a} \cap I_{b}$ while if $a_{i} \in I_{a} \cap I_{b}$, then $a_{i} \geq a, b$ and thus $a_{i} \geq a \vee b$ so that $I_{a} \cap I_{b} \subseteq I_{a \vee b^{*}}$. This means that

$$
r(a \vee b)=i \backslash\left(I_{a} \cap I_{b}\right)=\left(I \backslash I_{a}\right) \cup\left(I \backslash I_{b}\right)=r(a) \cup r(b)
$$

Suppose that for some $a, b \in J$ with $a \neq b$, we have $r(a)=r(b)$. We may suppose that $b \nless a$ so that $a \vee b>a$. Then, by the above, $r(a \vee b)=r(a) \cup r(b)=\tau(a)$. Thus $I_{a \vee b}=I_{a^{*}}$. But since $\omega(a \vee b)>\omega(a),\left|I_{a \vee b}\right|<\left|I_{a}\right|$, and we have a contradiction. Thus $r$ is a semilattice monomorphism from $J$ to $B(I)$. We calculate that for $a \in J$

$$
|r(a)|=|I|-\left|I_{a}\right|=|I|-(r-\omega(a))=\omega(a)-(r-|I|),
$$

where $r-|I| \geq 0$. Let $t=r-|I|$ and let $I^{*}$ be a $t$ element set with $I \cap I^{*}=\varnothing$. Then $\left|I \cup I^{*}\right|=r$ and $\sigma: J \rightarrow B\left(I \cup I^{*}\right)$ defined by $\sigma(a)=r(a) \cup I^{*}$ is a semilattice isomorphism with $|\sigma(a)|=|\tau(a)|+t=\omega(a)$. This completes the proof of the theorem.

3. Application to transversal matroids. A characterization of transversal matroids is given by Brualdi and Dinolt [3]. We shall use the result of $\$ 2$ to give an alternate formulation of it. But first we review briefly matroids, in general, and transversal matroids, in particular; for further details we refer the reader to [3] and the references contained within.

Let $E$ be a finite set. A matroid [5] M on $E$ (or combinatorial pregeometry [4]) is a nonempty collection of subsets of $E$, called independent sets such that 
(i) a subset of an independent set is independent (thus $\varnothing \in \mathrm{M}$ ) (ii) $A_{1}, A_{2} \in \mathrm{M}$ with $\left|A_{1}\right|<\left|A_{2}\right|$ imply $A_{1} \cup\{x\} \in M$ for some $x \in A_{2} \backslash A_{1}$. Each subset $X$ of $E$ has a well-defined rank $\rho(X)$ which equals the common cardinality of all maximal independent sets contained in $X$. The rank of the matroid $M$ equals $\rho(E)$. For $X \subseteq E, M_{X}=\{A: A \in M, A \subseteq X\}$ is a matroid, called the restriction of $M$ to $A$. A closure relation can be defined on the subsets of $E$ by defining $\bar{X}$ to be the largest subset of $E$ containing $X$ which has the same rank as $X$. Those subsets $F$ of $E$ with $\bar{F}=F$ are called flats. The collection $\mathcal{L}(M)$ of flats of $M$, partially ordered by set-theoretic inclusion, form a geometric lattice [4]. If $X \subseteq E$, then $x \in X$ is a coloop or isthmus of $X$ if $\rho(X \backslash\{x\})=\rho(X)-1$. The collection $\mathcal{F}(\mathbf{M})$ of flats of M which have no coloops forms a join-subsemilattice of $\mathcal{L}(M)$. Given a pair $F_{1}$, $F_{2}$ of flats in $\mathscr{F}(M)$ with $F_{1} \subseteq F_{2}$, such that no other flat of $\mathscr{F}(M)$ lies between $F_{1}$ and $F_{2}$, then the interval $\left[F_{1}, F_{2}\right]$ of $\mathscr{L}(M)$ consists of all sets of the form $F_{1} \cup A$ where $A \subseteq F_{2} \backslash F_{1},|A| \leq \rho\left(F_{2}\right)-\rho\left(F_{1}\right)-1$, along with $F_{2}$. Thus the flats of $\mathcal{F}(M)$, given as subsets of $E$ with the ir rank, determine all flats of $\mathscr{L}(M)$ as sets and thus the partial order of $\mathscr{L}(M)$; that is, they determine the lattice $\mathcal{L}(\mathrm{M})$.

A matroid $M$ on $E$ is a transversal matroid provided there is a family ( $A_{1}$, $\left.\cdots, A_{n}\right)$ of subsets of $E$ such that $\mathrm{M}=\mathrm{M}\left(A_{1}, \cdots, A_{n}\right)$, the collection of partial transversals of $\left(A_{1}, \cdots, A_{n}\right)$. If $M$ is a transversal matroid of rank $r$, then there are $r$ sets $A_{1}, \cdots, A_{r}$ such that $\mathrm{M}=\mathrm{M}\left(A_{1}, \cdots, A_{r}\right)$. The family $\left(A_{1}, \cdots, A_{r}\right)$ is called a presentation of $M$. We recall Hall's theorem which says that the family $\left(A_{1}, \cdots, A_{r}\right)$ has a transversal (thus a system of distinct representatives) if and only if

$$
\left|\bigcup_{i \in K} A_{i}\right| \geq|K| \quad(K \subseteq\{1, \cdots, r\})
$$

Now let $M$ be an arbitrary matroid of rank $r$ on the finite set $E$. We regard the join-semilattice $\mathcal{F}(M)$ as a weighted join-semilattice by letting $\omega(F)=\rho(F)$ for $F \in \mathcal{F}(M)$. The unique flat in $\mathscr{F}(M)$ of weight 0 is the closure of the empty set. In the terminology of $\$ 2$ the characterization of transversal matroids given in [3] is the following: $M$ is a transversal matroid if and only if $\mathcal{F}(M)$ has a spread $\left(F_{1}, \cdots, F_{r}\right)$ where $\rho\left(\bigcap_{i \in K} F_{i}\right) \leq r-|K|(K \subseteq\{1, \cdots, r\})$. It is also proved in $[3]$ that if $M$ is a transversal matroid, then $M=M\left(E \backslash F_{1}, \cdots, E \backslash F_{r}\right)$; indeed $\left(E \backslash F_{1}, \cdots, E \backslash F_{r}\right)$ is the maximal presentation of $M$. This means that we cannot enlarge any of the sets $E \backslash F_{1}, \cdots, E \backslash F_{r}$ and still have a presentation of $M$. It is enough to know that the sets $F_{1}, \cdots, F_{r}$ have no coloops, in order to conclude that $\left(E \backslash F_{1}, \cdots, E \backslash F_{r}\right)$ is the maximal presentation of $M$ ([1] and [3]). 
If $G_{1}, G_{2}$ are flats in $\mathcal{L}(\mathrm{M})$ with $G_{1} \subseteq G_{2}$, then $\mathcal{F}(\mathrm{M})\left[G_{1}, G_{2}\right]$ denotes the join-subsemilattice of $\mathscr{L}(M)$ consisting of all flats of $\mathscr{L}(M)$ with no coloops which lie in the interval $\left[G_{1}, G_{2}\right]$ of $\mathscr{L}(M)$. Note that $\mathcal{F}(M)=\mathscr{F}(M)[\varnothing, E]$, and that $\mathcal{F}(M)_{\left[G_{1}, G_{2}\right]}$ is a join-subsemilattice of $\mathcal{F}(M)$. We regard $\mathcal{F}(M)_{\left[G_{1}, G_{2}\right]}$ as weighted by rank (or we could assign $F \in \mathcal{F}(M)_{\left[G_{1}, G_{2}\right.}$ the weight $\rho(F)-\rho\left(G_{2}\right)$ ).

Theorem 3.1. Let $M$ be a matroid of rank $r$ on the finite set $E$. Then $M$ is a transversal matroid if and only if for all $G_{1}, G_{2} \in \mathscr{L}(\mathrm{M})$ with $G_{1} \subseteq G_{2}$ the weighted join-semilattice $\mathcal{F}(M)_{\left[G_{1}, G_{2}\right]}$ bas a spread of at most $\rho\left(G_{2}\right)-\rho\left(G_{1}\right)$ members or, equivalently, there is a weight-preserving join-semilattice isomorphism from $\mathcal{F}(\mathrm{M})_{\left[G_{1}, G_{2}\right]}$ to a Boolean lattice on an $\rho\left(G_{2}\right)-\rho\left(G_{1}\right)$ element set.

Proof. By Theorem 2.4 the two criteria are equivalent. Suppose first that $M$ is a transversal matroid of rank $r$. Then $\mathcal{F}(M)$ has a spread $\left(F_{1}, \cdots, F_{r}\right)$. Let $G \in \mathcal{L}(\mathrm{M})$ with $\rho(G)=r-k$. Then those members of $\left(F_{1}, \cdots, F_{r}\right)$ which conta in $G$ are the members of a spread of $\mathcal{F}(M)_{[G, E]}$. Let this spread be $\left(F_{k}: k \in K\right)$ where $K \subseteq\{1, \cdots, r\}$. Since $\rho\left(\bigcap_{i \in K} F_{i}\right) \leq r-|K|$ and since $G \subseteq \bigcap_{i \in K} F_{i}$ we have $\rho(G)=r-k \leq \rho\left(\bigcap_{i \in K} F_{i}\right)$, and we conclude that $|K| \leq k$. Thus $\mathscr{F}(\mathbb{M})_{[G, E]}$ has a spread of at most $k$ members where $k=\rho(E)-\rho(G)$. Since $M_{G_{2}}$ is a transversal matroid of rank $\rho\left(G_{2}\right)$ on $G_{2}$ and $\mathscr{F}(\mathrm{M})\left[G_{1}, G_{2}\right]$ is isomorphic to $\mathcal{F}\left(\mathrm{M}_{G_{2}}\right)_{\left[G_{1}, G_{2}\right]}$, we conclude that $\mathcal{F}(\mathrm{M})_{\left[G_{1}, G_{2}\right]}$ has a spread of at most $\rho\left(G_{2}\right)$ $\rho\left(G_{1}\right)$ members for any $G_{1}, G_{2} \in \mathcal{L}(\mathrm{M})$ with $G_{1} \subseteq G_{2}$.

Suppose now $M$ is a matroid of rank $r$ such that for all $G \in \mathscr{L}(M), \mathcal{F}(M)_{[G, E]}$ has a spread of at most $r-\rho(G)$ members. Thus, in particular, $\mathcal{F}(M)$ has a spread $\left(F_{1}, \cdots, F_{r}\right)$ with $r$ members. Suppose for some $K \subseteq\{1, \cdots, r\}, \rho\left(\bigcap_{i \in K} F_{i}\right)>r-$ $|K|$. Let $G=\bigcap_{i \in k} F_{i}$. Then $\mathcal{F}(M)_{[G, E]}$ has a spread with at most $r-\rho(G)$ members. But a spread of $\mathcal{F}(M)_{[G, E]}$ consists of all members of the spread of $\mathcal{F}(M)$ which contain $G$; thus $F_{i}(i \in K)$ are members of the spread of $\mathcal{F}(M)_{[G, E]}$. We conclude that $|K| \leq r-\rho(G)$ or $\rho(G) \leq r-|K|$, and this is a contradiction. Hence $\rho\left(\bigcap_{i \in K} F_{i}\right) \leq r-|K|(K \subseteq\{1, \cdots, r\})$ and $M$ is a transversal matroid.

We mention one application to an interesting class of matroids. Let $E$ be a set and $\left\{X_{i}: 1 \leq i \leq k\right\}$ a collection of subsets of $E$ such that (i) $\left|X_{i}\right| \geq r-1 \quad(1 \leq i \leq k)$ and (ii) every $r-1$ element subset of $E$ is a subset of exactly one of $X_{1}, \cdots, X_{k}$. Then [4] the set $E$, the $X_{i}(1 \leq i \leq k)$, and all subsets $A$ of $E$ with $|A| \leq r-2$ are the flats of a geometry (therefore matroid $M$ ) on $E$ of rank $r$. Such a geometry is called a Hartmanis geometry [4]. In this case $\mathcal{F}(M)$ consists of those $X_{i}$ with $\left|X_{i}\right| \geq r$ (these are flats of rank $r-1$ ), $\varnothing$, and possibly $E$. Thus $\mathcal{F}(M)$ has a 
spread if and only if $|J| \leq r$ where $J=\left\{i: 1 \leq i \leq k,\left|X_{i}\right| \geq r\right\}$. The spread is then $\left(X_{i}: i \in J\right)$ along with the $\varnothing$ with the correct multiplicity to give $r$ sets in total.

Theore $\mathrm{m}$ 3.2. The Hartmanis geometry $\mathbf{M}$ is a transversal geometry if and only if $\left|\bigcap_{i \in I} X_{i}\right| \leq r-|I|(I \subseteq J,|I| \geq 2)$.

If $I \subseteq J,|I| \geq 2$, then $\rho\left(\bigcap_{i \in I} X_{i}\right)=\left|\bigcap_{i \in I} X_{i}\right|$.

4. Construction of transversal matroids. Let $M$ be a transversal matroid of rank $r$ on a finite set $E$, and let $\mathcal{F}(M)$ be the join-semilattice of flats with no coloops, weighted by rank. Then we know there is a join-subsemilattice $J$ of the Boolean lattice on an $r$ element set such that $\mathcal{F}(M)$ and $J$ are isomorphic as weighted join-semilattices. Since $\mathcal{F}(M)$ has a minimal element $\bar{\phi}, \mathcal{F}(M)$ is a lattice. (Note, however, $\mathcal{F}(M)$ is not in general a sublattice of $\mathscr{L}(M)$; it is, however, a join-subsemilattice of $\mathcal{L}(M)$.) We consider the following question. Suppose $J$ is a join-subsemilattice with minimal element of the Boolean lattice on an $r$ element set, weighted by cardinality. Is there a transversal matroid $M$ of rank $r$ such that $\mathcal{F}(\mathrm{M})$ and $J$ are isomorphic as we ighted join-semilattices?

Theorem 4.1. Let $J$ be a join-subsemilattice of a Boolean lattice on an $r$ element set, weighted by cardinality, such that $0,1 \in J$ with $\omega(0)=0, \omega(1)=r$. Then there is a transversal matroid $\mathbf{M}$ of rank $r$ on a finite set $E$ such that $\mathcal{F}(\mathbf{M})$ and $J$ are isomorpbic as weighted partially ordered sets; that is, there is a bijection $\sigma: J \rightarrow \mathcal{F}(\mathrm{M})$ sucb that

$$
a<b \text { if and only if } \sigma(a)<\sigma(b) \quad(a, b \in J),
$$

$$
|a|=|\omega(\sigma(a))| \quad(a \in J)
$$

We shall devote the remainder of this section to proving this theorem. The proof will be divided into several parts, but first we need a construction.

Let $E^{\prime}$ be some sufficiently large set. Corresponding to each $a \in J$ we define a subset $F_{a}$ of $E^{\prime}$ as follows:

(0) $F_{0}=\varnothing$.

(1) If $a \in J$ with $\omega(a)=1$, choose distinct elements $x, y$ of $E^{\prime}$ and set $F_{a}=$ $\{x, y\}$. We do this for each $a \in J$ of weight 1 in such a way that all elements chosen are distinct: $F_{a} \cap F_{b}=\varnothing$ if $a, b \in J, \omega(a)=\omega(b)=1, a \neq b$.

(k) If $a \in J$ with $\omega(a)=k$, let $J_{a}=\{x \in J: x<a\}$. For each $x \in J_{a}, \omega(x)<k$. If $a=\bigvee\left\{x: x \in J_{a}\right\}$, set $F_{a}=\bigcup\left\{F_{x}: x \in J_{a}\right\}$. If $\bigvee\left\{x \in J_{a}\right\}=b<a$ and $\omega(b)=$ $l<k$, then choose a subset $X_{a}$ of $E^{\prime}$ with $\left|X_{a}\right|=k-l+1$ where the elements of $X_{a}$ are different from any chosen previously. Then set $F_{a}=F_{b} \cup X_{a}$. We do 
this for each element of $J$ of weight $k$ in such a way that $X_{a_{1}} \cap X_{a_{2}}=\varnothing$ whenever $\omega\left(a_{1}\right)=\omega\left(a_{2}\right)=k$ and $a_{1} \neq a_{2}$.

$\vdots$

The construction ends after we have gone through all elements of $J$. The family of sets $\mathcal{F}=\left(F_{a}: a \in J\right)$ obtained is partially ordered by set-theoretic inclusion. Let $E=\bigcup_{a \in J} F_{a}$.

$$
a \leq b \text { if and only if } F_{a} \subseteq F_{b} \quad(a, b \in J) .
$$

Thus $a \neq b$ implies $F_{a} \neq F_{b}$, and the partially ordered sets $\mathcal{F}$ and $J$ are isomorphic.

By construction it is clear that if $a \leq b$ then $F_{a} \subseteq F_{b}$. We need to prove conversely that $F_{a} \subseteq F_{b}$ implies $a \leq b$, and we do this by induction on weight. It is certainly true by construction if $a$ and $b$ have weight at most 1 . Let $k>1$ and ass ume that $F_{a} \subseteq F_{b}$ implies $a \leq b$ if $\omega(a)<k, \omega(b)<k$. Now consider $a$, $b \in J$ with $\omega(a) \leq k, \omega(b) \leq k$. We may assume by the induction that one of the latter is an equality.

We first make the following observation. For $x \in E$ let $\beta(x)$ be the element of $J$ such that $x \in X_{\beta(x)^{\circ}}$. Thus in the construction $x$ makes its first appearance in the set $F_{\beta(x)}$. It then follows for $x \in E$ and $c \in J$ that $\beta(x) \leq c$ if and only if $x \in F_{c}$.

Now if $a \neq \bigvee_{x<a^{x}}$, then $X_{a} \neq \varnothing$. Let $z \in X_{a}$. Since $F_{a} \subseteq F_{b}, z \in F_{b}$; hence $a=\beta(z) \leq b$. Thus we may assume $a=\bigvee_{x<a}$. Let $p=\bigvee_{z \in F} \beta(z)$. Thus $F_{p}=F_{a}$. Since $z \in F_{a}$ also implies $z \in F_{b}, \beta(z) \leq a, b$ for $z \in F_{a}$ and hence $p \leq a \wedge b$. If $p=a$, then $a \leq b$. If $p<a$, then consider $x \in J$ with $x<a$. We have $F_{x} \subseteq F_{p}$. Since $\omega(x), \omega(p)<\omega(a)=k$, we have by induction that $x \leq p$. Hence $a=\bigvee_{x<a} \leq p$. Since $p \leq a$, this implies $a=p$, a contradiction. Thus $a=p$ and $a \leq b$.

(4.1.4) The meet operation in the lattice $\mathcal{F}$ is set-theoretic intersection.

Let $a, b \in J$ and $c=a \wedge b$, so that $F_{c}=F_{a} \wedge F_{b}$. Then $F_{c} \subseteq F_{a} \cap F_{b}$. Suppose there were an $x \in\left(F_{a} \cap F_{b}\right) \backslash F_{c} ;$ thus $\beta(x) \leq a, b$ so that $\beta(x) \leq a \wedge$ $b=c$. This means $x \in F_{c}$, which is a contradiction.

We let the isomorphism $\sigma: J \rightarrow \mathcal{F}$ where $\sigma(a)=F_{a}$ carry over the weight function of $J$ to $\mathcal{F}$. That is, we define $\omega\left(F_{\dot{a}}\right)=\omega(a)(a \in J)$. Since $J$ is a joinsubsemilattice of the Boolean lattice of an $r$ element set with 0 and 1 , weighted by cardinality, $J$ has a spread $\left(a_{i}: 1 \leq i \leq r\right)$. Thus $\left(F_{a_{i}}: 1 \leq i \leq r\right)$ is the spread of $\mathcal{F}$. Consider the transversal matroid $M=M\left(E \backslash F_{a_{1}}, \cdots, E \backslash F_{a_{r}}\right)$. We have several things to verify concerning $\mathcal{F}$ and the matroid $M$. 


$$
\text { If } a>b \text {, then }\left|F_{a} \backslash F_{b}\right| \geq \omega(a)-\omega(b)+1 \geq 2 \text {. }
$$

To prove this we apply induction to $\omega(a)$. If $\omega(a)=1$ then $\omega(b)=0$ and by construction $F_{b}=\varnothing,\left|F_{a}\right| \geq 2$. Thus assume $\omega(a)=k$ and that the result holds when the weight is less than $k$. If there is $c \in J$ such that $a>c>b$, then by induction $\left|F_{c} \backslash F_{b}\right| \geq \omega(c)-\omega(b)+1$. Thus if $\left|F_{a} \backslash F_{c}\right| \geq \omega(a)-\omega(c)+1$ then $\left|F_{a} \backslash F_{b}\right| \geq \omega(a)-\omega(b)+2$. Thus we might as well assume that there is no such c. If $x<a$ implies $x \leq b$, then $\bigvee\{x: x<a\} \leq b$. Thus by construction $\left|F_{a} \backslash F_{b}\right|=$ $\omega(a)-\omega(b)+1$. Otherwise there is an $x<a$ such that $x \underline{k} b$. Then $\omega(x \wedge b)<$ $\omega(x)<k$ so that by induction $A=F_{x} \backslash F_{x \wedge b}=F_{x} \backslash\left(F_{x} \cap F_{b}\right)$ has cardinality at least $\omega(x)-\omega(x \wedge b)+1$. But since $J$ is a join-subsemilattice of a Boolean lattice and weighted by cardinality, $\omega(x \vee b)+\omega(x \wedge b) \leq \omega(x)+\omega(b)$. Since $b<x \vee b \leq a$, we have $a=x \vee b$. Thus $\omega(a)-\omega(b) \leq \omega(x)-\omega(x \wedge b)$. Since $A \subseteq F_{a} \backslash F_{b}$ and $|A| \geq \omega(a)-\omega(b)+1$, we are done.

$$
\text { The family }\left(E \backslash F_{a_{1}}, \cdots, E \backslash F_{a_{r}}\right) \text { has a transversal. }
$$

We need to show that the condition for the existence of a transversal is satisfied here. We calculate that for $\varnothing \neq K \subseteq\{1, \cdots, r\}$

$$
\left|\bigcup_{i \in K} E \backslash F_{a_{i}}\right|=\left|E \backslash \bigcap_{i \in K} F_{a_{i}}\right|=|E|-\left|F_{z}\right|
$$

where $z=\bigwedge\left\{a_{i}: i \in K\right\}$. But since $\left(a_{i}: 1 \leq i \leq r\right)$ is a spread of $J, \omega\left(\bigwedge_{i \in K} a_{i}\right) \leq$ $r-|K|$; otherwise we contradict the definition of a spread. Thus $\omega(z) \leq r-|K|$. If we apply (4.1.5) with $a=1\left(F_{1}=E\right)$ and $b=z$, we have

$$
\left|E \backslash F_{z}\right| \geq r-\omega(z)+1 \geq r-(r-|K|)+1=|K|+1 .
$$

Thus we have a transversal.

$$
\text { For } a \in J, F_{a} \text { is a flat of } M \text {. }
$$

Let $\omega(a)=r-k$. Then exactly $k$ members of the spread $\left(a_{i}: 1 \leq i \leq r\right)$, say $a_{1}, \cdots, a_{k}$, satisfy $a_{i} \geq a(i=1, \cdots, k)$ and $a=\Lambda\left(a_{i}: 1 \leq i \leq k\right)$. Since $\mathcal{F}$ is lattice isomorphic to $J$ via $\sigma(a)=F_{a}, F_{a}=\bigcap\left(F_{a_{i}}: 1 \leq i \leq k\right)$. Thus

$$
F_{a} \cap\left(E \backslash F_{a_{i}}\right)=\varnothing \quad(1 \leq i \leq k)
$$

Now let $x \in E \backslash F_{a^{*}}$. Thus $x \in \bigcup_{i=1}^{k}\left(E \backslash F_{a_{i}}\right)_{0}$ If $B$ is a maximum partial transversal contained in $F_{a}$ (thus the rank of $F_{a}{ }_{a}$ in $M$ is $|B|$ ), then $B \cup x$ is also a partial transversal. Thus $F$ is closed and therefore a flat of $M$. 
(4.1.8) If $a \in J$ has weight $r-k$, then the flat $F_{a}$ of $M$ has rank equal to $r-k$. Thus the rank function coincides with the weight function on $\mathfrak{F}$.

Since $\omega(a)=r-k$, there are exactly $k$ members of $\left(a_{i}: 1 \leq i \leq r\right)$, say $a_{1}$, $\cdots, a_{k}$, which are greater than or equal to $a$. Thus $F_{a} \subseteq F_{a_{i}}(1 \leq i \leq k)$. Consider the family $\left(\left(E \backslash F_{a_{i}}\right) \cap F_{a}: k+1 \leq i \leq r\right)$ of subsets of $F_{a}$. We show that this family has a transversal which will prove $\rho(F)=r-k$. Let $K \subseteq\{k+1, \cdots, r\}$. Then

$$
\left|\bigcup_{i \in K}\left(E \backslash F_{a_{i}}\right) \cap F_{a}\right|=\left|\left(E \backslash \bigcap_{i \in K} F_{a_{i}}\right) \cap F_{a}\right|=\left|F_{a} \backslash \bigcap_{i \in K}\left(F_{a_{i}} \cap F_{a}\right)\right| .
$$

Let $\bigcap_{i \in K} F_{a_{i}} \cap F_{a}=F_{b}$. Since $F_{a}=\bigcap_{i=1}^{k} F_{a_{i}}$, at least $k+|K|$ members of the spread $\left(F_{a_{i}}: 1 \leq i \leq r\right)$ of $\mathcal{F}$ contain $F_{b}$. Thus $\omega\left(F_{b}\right) \leq r-(k+|K|)$, and so by $(4.1 .5)$

$$
\left|F_{a} \backslash F_{b}\right| \geq \omega(a)-\omega(b)+1 \geq r-k-(r-(k+|K|))+1=|K|+1 .
$$

Thus the defined family has a transversal, which proves the statements made.

For $a \in J$, the flat $F_{a}$ of $M$ has no coloops.

We prove this by induction on $\rho\left(F_{a}\right)=\omega\left(F_{a}\right)$. If $\rho\left(F_{a}\right)=0$ or 1 , this is true by construction. Let $\rho\left(F_{a}\right)=k>1$, and assume the result is true for rank smaller than $k$. Suppose first $a=\bigvee_{x<a}$. Then $F_{a}=\bigvee_{x<a} F_{x}$ and by induction each $F_{x}$ is a flat of $M$ with no coloops. Since the join of flats with no coloops of a matroid has no coloops, this proves $F_{a}$ has no coloops. Suppose now $V_{x<a} x=$ $b<a$ and thus $\rho\left(F_{b}\right)<k$. Since by induction $F_{b}$ has no coloops, no element of $F_{b}$ can be a coloop of $F_{a}$. But $F_{a}=F_{b} \cup X_{a}$ where $\left|X_{a}\right|=\rho\left(F_{a}\right)-\rho\left(F_{b}\right)+1$. Thus if $B$ is a maximal independent set of $M$ contained in $F_{b}$, then $B \cup\left(X_{a} \backslash x\right)$ is a maximal independent set contained in $F_{a}$. Thus no element of $X_{a}$ is a coloop of $F_{a}$, and $F_{a}$ has no coloops.

Finally we show

(4.1.10) If $F$ is a flat of $M$ with no coloops, then $F=F_{a}$ for some $a \in J$.

Consider a flat $F$ of $M$ with no coloops and let $\rho(F)=r-k$. Since $M=$ $\mathrm{M}\left(E \backslash F_{a_{1}}, \cdots, E \backslash F_{a_{r}}\right)$ where $F_{a_{i}}$ is a flat with no coloops of $M(1 \leq i \leq r)$, then $\left(E \backslash F_{a_{1}}, \cdots, E \backslash F_{a_{r}}\right)$ is the maximal presentation of $M$. Thus $\left(F_{a_{1}}, \cdots, F_{a_{r}}\right)$ is the spread of $\mathcal{F}(M)$ (recall it was defined to be the spread of $\mathfrak{F}$ ). Thus since $\rho(F)=r-k$ there are exactly $k$ members of $\left(F_{a_{1}}, \cdots, F_{a_{r}}\right)$, say $F_{a_{1}}, \cdots, F_{a_{k}}$ 
which contain $F$. Thus $F=\bigcap_{i=1}^{k} F_{a_{i}}$ But by (4.1.4) $\bigcap_{i=1}^{k} F_{a_{i}}=F_{b}$ for some $b \in J$. Thus $F=F_{b}$.

This now completes the proof of Theorem 4.1.

\section{REFERENCES}

1. G. Birkhoff, Lattice theory, 3rd ed., Amer. Math. Soc. Colloq. Publ., vol. 25, Amer. Math. Soc., Providence, R. I., 1967. MR 37 \#2638.

2. J. A. Bondy and D. J. A. Welsh, Some results on transversal matroids and constructions for identically self-dual matroids, Quart. J. Math. Oxford Ser. (2) 22 (1971), 435-451. MR 44 \#3899.

3. R. A. Brualdi and G. W. Dinolt, Characterizations of transversal matroids and their presentations, J. Combinatorial Theory 12 (1972), 268-286.

4. H. Crapo and G. C. Rota, On the foundations of combinatorial theory: Combinatorial geometries, Preliminary edition, M. I. T. Press, Cambridge, Mass., 1970. MR 45 $\# 74$.

5. H. Whitney, On the abstract properties of linear dependence, Amer. J. Math. 57 (1935), 509-533.

DEPARTMENT OF MATHEMATICS, UNIVERSITY OF WISCONSIN, MADISON, WISCONSIN 53706 